Check for updates

Cite this: Chem. Commun., 2019, 55,12479

Received 12th September 2019,

Accepted 24th September 2019

DOI: $10.1039 / \mathrm{c} 9 \mathrm{cc} 07129 f$

rsc.li/chemcomm

\section{A novel electrochemiluminescent emitter of europium hydroxide nanorods and its application in bioanalysis $\dagger$}

\author{
Chunyuan Tian, ${ }^{a}$ Li Wang, ${ }^{a}$ Feng Luan, ${ }^{a}$ Xiuli Fu, ${ }^{a}$ Xuming Zhuang (D) *a and \\ Lingxin Chen iD *bcd
}

The electrochemiluminescence (ECL) from europium hydroxide nanorods (EHNs) was reported for the first time using $\mathrm{K}_{2} \mathrm{~S}_{2} \mathrm{O}_{8}$ as an efficient cathodic co-reactant, and an ultrasensitive ECL aptasensor based on the system for the detection of thrombin has been formulated successfully.

Recently, semiconductor quantum dot (QD)-based ECL has been attracting much attention in the field of bioanalysis, due to the unique optical and electrochemical properties of luminescent nanocrystals. ${ }^{1-3}$ Among them, semiconductor QDs such as CdSe, ${ }^{4,5}$ $\mathrm{CdS},{ }^{6,7}$ and $\mathrm{PdS}^{8,9}$ have been widely chosen as ECL emitters, which can be ascribed to their narrow emission band, good photostability, and high quantum yields. However, the frequent use of conditional semiconductor nanocrystals, which contain heavy metals (such as $\mathrm{Cd}^{2+}$ and $\mathrm{Pb}^{2+}$ ), as the basic elements of ECL emitters can cause environmental and health hazards due to their high toxicity and poor biodegradability. Thus, a good deal of attention has been paid to the development of new environment-friendly and low-toxicity or nontoxic ECL emitters for bioassays. ${ }^{10-14}$

As is well known, rare earth nanomaterials have been used in many fields due to their excellent magnetic and optical properties, controllable structure and excellent biocompatibility. ${ }^{15}$ The related research on rare earth compound nanocrystals is also now rapidly developing, and many kinds of nanomaterials have been reported, such as oxides, ${ }^{16,17}$ hydroxides, ${ }^{18}$ fluorides, ${ }^{19}$ phosphates, ${ }^{20,21}$ and sulfides, ${ }^{22}$ which have received tremendous

\footnotetext{
${ }^{a}$ College of Chemistry and Chemical Engineering, Yantai University, Yantai 264005, China. E-mail: xmzhuang@iccas.ac.cn

${ }^{b}$ CAS Key Laboratory of Coastal Environmental Processes and Ecological Remediation, Yantai Institute of Coastal Zone Research, Chinese Academy of Sciences, Yantai 264003, China. E-mail: lxchen@yic.ac.cn

${ }^{c}$ Laboratory for Marine Biology and Biotechnology, Pilot National Laboratory for Marine Science and Technology, Qingdao 266237, China

${ }^{d}$ Center for Ocean Mega-Science, Chinese Academy of Sciences, Qingdao 266071, China

$\dagger$ Electronic supplementary information (ESI) available: Materials and apparatus. Preparation of $\mathrm{Eu}(\mathrm{OH})_{3}$ nanorods. Preparation of EHNs/GCE film. Fabrication of CdTe/MNPs. Preparation of ssDNA/CdTe/MNP composites. Fabrication of the ECL aptasensor. See DOI: 10.1039/c9cc07129f
}

attention due to their specific properties arising from the $4 \mathrm{f}$ electron configuration of the lanthanides. As a family of europium compound nanomaterials, europium hydroxide nanorods (EHNs) have been widely studied and applied to tissue engineering and related applications due to their good biocompatibility and low cytotoxicity. ${ }^{23-25}$ As far as we know, there has been no research reported on the possibility of using EHNs as ECL emitters in an ECL biosensing platform.

The experimental test results indicate that highly stable and repeatable ECL emission can be generated on an EHN-modified glassy carbon electrode (EHNs/GCE), which will be conducive to use in accurate bioanalysis. Thrombin (TB), a serine protease with multiple functions, is the terminal enzyme in the coagulation cascade, and could be involved in a number of physiological and pathological processes, such as thrombosis, hemostasis, incrustation and inflammation. ${ }^{26,27}$ The aim of the present study is to verify the application of an EHNs/GCE as an efficient ECL emitter in bioanalysis. An aptamer-based target assay was fabricated using TB as a model.

The magnetic nanoparticle-loaded CdTe nanoparticles, denoted as CdTe/MNPs, were prepared by hydrothermal synthesis following the literature method. ${ }^{28}$ The efficient quenching of CdTe/MNPs has been well documented, which was ascribed to the CdTe/MNPs as a black-body-like material efficiently absorbing ECL emission from the EHNs/GCE. On the basis of this, and to specifically recognize its aptamer and $\mathrm{TB}$, an aptasensor for the detection of TB has been constructed. The fabrication procedure of the ECL biosensor is illustrated in Scheme 1. When the quenching probe ssDNA/CdTe/MNPs was connected to the EHNs/GCE surface by hybridization between the aptamers and their complementary DNA (ssDNA), the ECL intensity was evidently decreased as a result of the quenching effect from the CdTe/MNPs. In the presence of target protein TB, the aptamer in the dsDNA on the electrode surface competitively combined with TB to form aptamer-TB bioaffinity complexes. The CdTe/ MNPs were removed from the electrode surface. Because the quenching efficiency of the ECL of the EHNs/GCE was determined by the amount of CdTe/MNPs bound to the dsDNA 

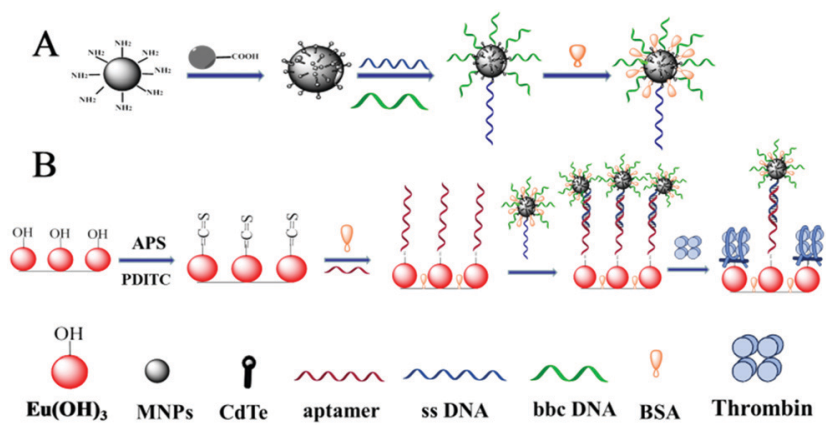

Scheme 1 (A) Schematic showing the preparation process of the CdTe/ MNP composites, and (B) schematic diagram of the construction of the ECL aptasensor for TB detection using the EHNs/GCE as an efficient ECL emitter.

oligonucleotides, it was inversely proportional to the combined target protein TB.

The EHNs were synthesized by using the hydrothermal method through the interaction of aqueous europium(III) nitrate $\left(\mathrm{Eu}\left(\mathrm{NO}_{3}\right)_{3}\right.$. $\left.6 \mathrm{H}_{2} \mathrm{O}\right)$ solution and sodium sulphide $\left(\mathrm{Na}_{2} \mathrm{~S} \cdot 9 \mathrm{H}_{2} \mathrm{O}\right)$ at $[\mathrm{Eu}: \mathrm{OH}]=$ $1: 2.5$ molar ratio in an open reflux system. Hydroxides were obtained by hydrolysis of $\mathrm{Na}_{2} \mathrm{~S}$, and similar reported examples are relatively rare in the preparation of EHNs. The morphology, structure and chemical composition of the product were confirmed by TEM, XRD, HRTEM, EDX and elemental mapping (Fig. 1a-d).

Fig. 1a, the TEM image of the ENHs, clearly illustrates that the as synthesized materials are entirely composed of nanorods with an approximate length of 150-200 nm and a diameter of $10 \mathrm{~nm}$. Compared with the conventional reaction, where EHNs are obtained by the reaction of aqueous europium nitrate hydrate with ammonium hydroxide, ${ }^{24,25}$ the method in this article proceeds at lower temperature and the nanorod products show higher aspect ratio. The XRD pattern of the as-synthesized ENHs (Fig. 1b) indicates the crystalline nature of the materials. The diffraction peaks of the nanorods are consistent with the standard data files (the JCPDS card No. 01-083-2305) for all reflections. ${ }^{29}$ The HRTEM image highlights that the EHNs possessed a well-defined crystalline structure with a lattice parameter of $0.305 \mathrm{~nm}$ along the (101) crystalline direction (Fig. 2c), consistent with the XRD data. The elemental mapping images of the EHNs were acquired as shown in Fig. 1d. It can be

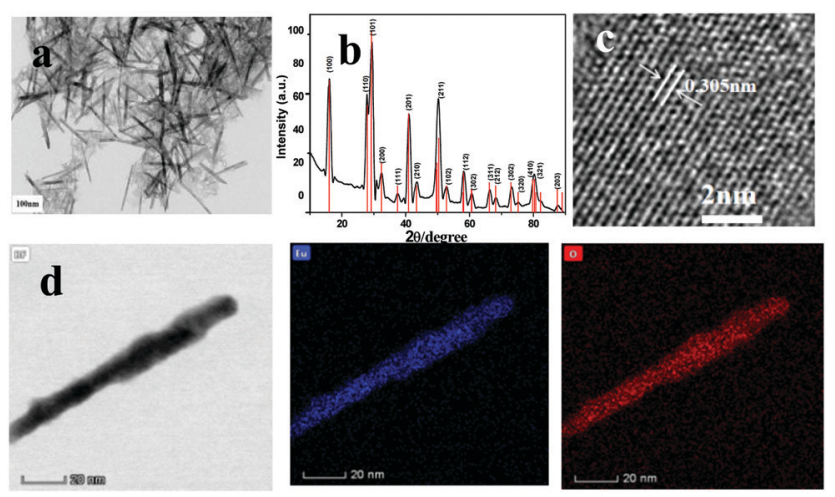

Fig. 1 Characterization of the ENHs: (a) HRTEM, (b) XRD, (c) TEM image of the ENHs and (d) STEM image and STEM-EDX elemental mapping of selected Eu and $\mathrm{O}$ elements.

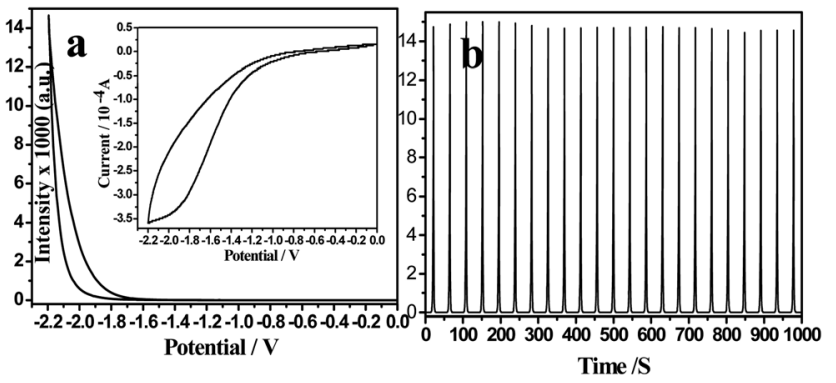

Fig. 2 (a) The ECL curves of the EHNs/GCE. Inset: The corresponding cyclic voltammograms. (b) ECL emission from the EHNs/GCE under continuous cyclic scans.

seen that the Eu and $\mathrm{O}$ elements were distributed throughout the nanostructure, which further confirmed the successful synthesis of EHNs.

The ECL behaviors of the EHNs/GCE in the presence of co-reactant $\mathrm{S}_{2} \mathrm{O}_{8}{ }^{2-}$ anions are shown in Fig. 2. As can be seen from the figure, the EHNs/GCE sustained high and stable ECL emission intensity. From the experimental results, the possible reaction mechanism of the ECL generated on the EHNs in $\mathrm{S}_{2} \mathrm{O}_{8}{ }^{2-}$ solution is outlined in the following equations:

$$
\begin{gathered}
\mathrm{EHNs}+n \mathrm{e} \rightarrow n \mathrm{EHNs}^{\bullet-} \\
\mathrm{S}_{2} \mathrm{O}_{8}{ }^{2-}+\mathrm{e} \rightarrow \mathrm{SO}_{4}{ }^{2-}+\mathrm{SO}_{4}{ }^{\bullet-} \\
\mathrm{EHNs}^{\bullet-}+\mathrm{SO}_{4}{ }^{\bullet-} \\
\rightarrow \mathrm{EHNS}^{*}+\mathrm{SO}_{4}{ }^{2-} \\
\mathrm{EHNs}^{*} \rightarrow \mathrm{EHNs}+h \nu
\end{gathered}
$$

In such a mechanism, EHNs*, the ECL-emitting species, was formed from the interaction of the co-reagent $\mathrm{K}_{2} \mathrm{~S}_{2} \mathrm{O}_{8}$. During the potential scanning with an initial negative direction in the ECL measurement process, the EHNs immobilized on the electrode were reduced to $\mathrm{EHNs}^{\bullet-}$ by charge injection. The reduction of $\mathrm{S}_{2} \mathrm{O}_{8}{ }^{2-}$ on the electrode produced a strong oxidant $\mathrm{SO}_{4}{ }^{\bullet-}$, and then EHNs ${ }^{\bullet-}$ could be oxidized by $\mathrm{SO}_{4}{ }^{\bullet-}$ to excited state EHNs* to emit light.

Fig. 2a inset displays the cyclic voltammograms (CVs) of the EHNs/GCE in $\mathrm{K}_{2} \mathrm{~S}_{2} \mathrm{O}_{8}$ solution, which showed a cathodic reduction current at a potential more negative than $-1.6 \mathrm{~V}$, which was mainly ascribed to the reduction of $\mathrm{K}_{2} \mathrm{~S}_{2} \mathrm{O}_{8}$ on the electrode surface.

Besides, the stability investigation of the EHNs/GCE was carried out under continuous cycling for 23 cycles with $0.05 \mathrm{M}$ $\mathrm{K}_{2} \mathrm{~S}_{2} \mathrm{O}_{8}$ as co-reagent. Fig. $2 \mathrm{~b}$ shows consecutive intensityconstant ECL peaks, suggesting that the ECL signal is extremely stable, which provides a reliable guarantee for the EHNs/GCE as a sensitive emitter for promising applications in biosensing.

TB is an important factor of the clotting system, and it plays a key role in the blood clotting cascade. In this experiment, TB was used as a model compound to illustrate the applicability of the EHNs/GCE working as an ECL emitter.

Fig. 3a displays the ECL intensity of the aptasensor with an increase in TB concentration varying from $0.01 \mathrm{fM}$ to $10 \mathrm{pM}$, and the intensity of the ECL signal was dependent on TB concentration. 

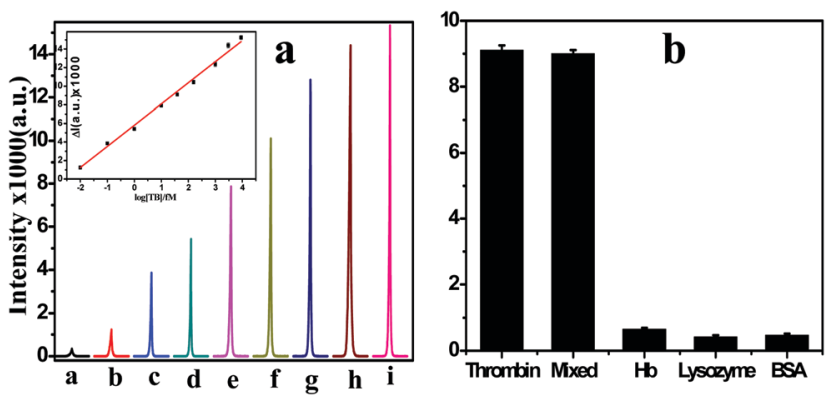

Fig. 3 (a) ECL signals of the aptasensor incubated with different concentrations of thrombin (a-i: 0, 0.01, 1, 10, 50, 100, 1000, 5000, and $10000 \mathrm{fM}$ ). Inset: Logarithmic calibration curve for thrombin. (b) Selectivity of the ECL proposed aptasensor to TB (0.1 pM) by comparing to interfering proteins at 1.0 pM: hemoglobin ( $\mathrm{Hb}$ ), lysozyme (Lyso), bovine serum albumin (BSA), and a mixed sample containing 0.1 TB and 1.0 pM BSA, Hb, and Lyso. Error bars represent RSD, $n=3$.

Fig. 3a inset shows the relationship between the changes in ECL signal $\left(\Delta I=I-I_{0}\right)$ and the logarithm of the TB concentration. The linear equation can be expressed as $\Delta I=2277.90 \log C_{\mathrm{TB}}+5587.56$ $(R=0.995)$, and the detection limit is detected as $0.01 \mathrm{fM}(\mathrm{S} / \mathrm{N}=3)$, where $I_{0}$ is the ECL intensity from the dsDNA/CdTe/MNPs/EHN electrode, and $I$ stands for ECL intensity after the electrode was incubated in TB solution. The proposed ECL aptasensor was compared with other previously reported ECL detection methods for $\mathrm{TB},{ }^{30-33}$ as shown in Table 1.

The selectivity of this prepared aptasensor was also tested under the same experimental conditions. As shown in Fig. 3b, when 1.0 pM BSA, Hb, and Lyso solutions were used to replace TB, the ECL intensity exhibits no obvious response, unlike when incubated in TB. However, the ECL intensity of the aptasensor was rapidly restored after being incubated in TB solution ( $0.1 \mathrm{pM})$ or a mixture is added. These results indicate that the constructed aptasensor has excellent selectivity for detecting TB.

In order to verify the practical applications of the prepared ECL aptasensor, the detection of TB in human serum samples was performed by sample recovery experiments. The results are shown in Table S1 (ESI $\dagger$ ). The recovery rates of TB (96.0-105.6\%) and the corresponding RSDs (4.2-5.7\%) are satisfactory within a reasonable error range, which clearly indicates the potential of this aptasensor for TB detection in real biological samples.

The inter-assay reproducibility of 6 as-constructed aptasensors was then investigated by detecting the same concentration of TB (1.0 pM). The inter-assay variation coefficient was $6.52 \%$,

Table 1 Comparison of the proposed electrode with other reported electrodes for the determination of TB

\begin{tabular}{llll}
\hline ECL emitter & LOD & Linear range & Ref. \\
\hline Pd NCs $^{a} /$ PAMAM $^{b}$ & $6.76 \mathrm{fM}$ & $0.01-100 \mathrm{pM}$ & 30 \\
GNPs-graphene $^{c}$ & $6.3 \mathrm{pM}$ & $0.01-10 \mathrm{nM}$ & 31 \\
PS $^{d}-(\mathrm{CdTe})_{2}$ & $0.35 \mathrm{pM}$ & $0.5-800 \mathrm{pM}$ & 32 \\
$\mathrm{CdS}$ & $26 \mathrm{aM}$ & $100 \mathrm{aM}-100 \mathrm{fM}$ & 33 \\
EHNs & $0.010 \mathrm{fM}$ & $0.01 \mathrm{fM}-10 \mathrm{pM}$ & This work
\end{tabular}

${ }^{a}$ Functional palladium nanocones. ${ }^{b}$ Amine-terminated polyamidoamine.

${ }^{c}$ Gold nanoparticle-graphene composite. ${ }^{d}$ Polystyrene microbeads. indicating that the reproducibility of the ECL aptasensor for TB determination was acceptable. It should be noted that the EHNs/GCE still exhibited over $82 \%$ activity towards 0.1 pM TB after being stored in the fridge for 10 days at $4{ }^{\circ} \mathrm{C}$.

In summary, a strong and stable ECL intensity from EHNs using $\mathrm{K}_{2} \mathrm{~S}_{2} \mathrm{O}_{8}$ as an efficient cathodic co-reactant has been reported for the first time. A new aptasensor system with the combination of the ECL behavior of EHNs and the excellent specificity of the aptamer technique has been formulated successfully. The aptasensor was applied for the sensitive and selective detection of TB with the detection limit down to $0.01 \mathrm{fM}$. Perfect results have been obtained while using the developed aptasensor to detect thrombin in actual human serum. This indicated that the EHNs with good biocompatibility provided alternative candidates to the toxic ECL emitters and may open promising avenues to develop new ECL systems for sensing assays.

The authors gratefully acknowledge the support of the National Natural Science Foundation of China (21405131, 21778047, 21675138, 21705139 and 21976209) and the National Natural Science Foundation of Shandong Province (ZR2017BB026).

\section{Conflicts of interest}

There are no conflicts to declare.

\section{Notes and references}

1 H. P. Huang, J.-J. Li and J.-J. Zhu, Anal. Methods, 2011, 3, 33-42.

2 W.-W. Zhao, J. Wang, Y. C. Zhu, J.-J. Xu and H. Y. Chen, Anal. Chem., 2015, 87, 9520-9531.

3 (a) Q. F. Zhai, J. Li and E. K. Wang, ChemElectroChem, 2012, 41, 5728-5743; (b) J. Yao, L. Li, P. F. Li and M. Yang, Nanoscale, 2017, 9, 13364-13383.

4 M. Amelia, C. Lincheneau, S. Silvi and A. Credi, Chem. Soc. Rev., 2012, 41, 5728-5743.

5 Y. P. Wei, X. P. Liu, C. J. Mao, H. L. Niu, J. M. Song and B. K. Jin, Biosens. Bioelectron., 2018, 103, 99-103.

6 M. S. Wu, H. W. Shi, J. J. Xu and H. Y. Chen, Chem. Commun., 2011, 47, 7752-7754.

7 Y. X. Hu, Y. Liu, S. Wang, Z. Y. Guo, Y. F. Hu and H. Z. Xie, ChemistrySelect, 2019, 4, 2926-2932.

8 M. Hesari, K. N. Swanick, J. S. Lu, R. Whyte, S. N. Wang and Z. F. Ding, J. Am. Chem. Soc., 2015, 137, 11266-11269.

9 H. F. Sha, Y. F. Wang, Y. Zhang, H. Ke, X. Xiong and N. Q. Jia, Sens. Actuators, B, 2018, 277, 157-163.

10 C. Y. Tian, L. Wang, F. Luan and X. M. Zhuang, Talanta, 2019, 191, 103-108.

11 B. Babamiri, A. Salimi and R. Hallaj, Biosens. Bioelectron., 2018, 117, 332-339.

12 M. Zhao, A. Y. Chen, D. Huang, Y. Zhuo, Y. Q. Chai and R. Yuan, Anal. Chem., 2016, 88, 11527-11532.

13 Y. Z. Wang, N. Hao, Q. M. Feng, H. W. Shi and H. Y. Chen, Biosens. Bioelectron., 2016, 77, 76-82.

14 X. Q. Chen, Y. Liu and Q. Ma, J. Mater. Chem., 2018, 6, 942-959.

15 Q. P. Li and B. Yan, J. Rare Earths, 2019, 7, 113-123.

16 T. Shen, Y. Zhang, A. M. Kirillov, B. Hu, C. Shan, W. Liu and Y. Tang, J. Mater. Chem. B, 2016, 4, 7832-7844.

17 J. Lin, S. S. Shi, J. Q. Zhang, Y. J. Zhang, L. Zhang, Y. Liu, P. P. Jin, P. F. Wei, R. H. Shi, W. Zhou and L. P. Wen, Small, 2016, 12, 5759-5768.

18 B. Lu, P. Cheng, H. B. Chen, Z. G. Sun and J. G. Pan, Rare Met. Mater. Eng., 2019, 48, 559-565.

19 X. Liu, R. Deng, Y. Zhang, Y. Wang, H. Chang, L. Huang and X. Liu, Chem. Soc. Rev., 2015, 44, 1479-1508. 
20 D. Qin and W. J. Tang, RSC Adv., 2017, 7, 2494-2502.

21 P. C. de Sousa Filho, E. Larquet, D. Dragoë, O. A. Serra and T. Gacoin, ACS Appl. Mater. Interfaces, 2017, 9, 1635-1644.

22 S. N. G. Corder, X. H. Chen, S. Q. Zhang, F. R. Hu, J. W. Zhang, Y. L. Luan, J. A. Logan, T. Ciavatti, H. A. Bechtel, M. C. Martin, M. Aronson, H. S. Suzuki, S. Kimura, T. Iizuka, Z. Fei, K. Imura, N. K. Sato, T. H. Tao and M. K. Liu, Nat. Commun., 2017, 8, 2262.

23 J. Z. Zhang, H. Q. Xie, Y. Shen, W. B. Zhao and Y. F. Li, Nanoscale, 2018, 10, 13617-13625.

24 R. Augustine, S. K. Nethi, N. Kalarikkal, S. Thomas and C. R. Patra, J. Mater. Chem., 2017, 5, 4660-4672.

25 P. F. Wei, L. Zhang, S. K. Nethi, A. K. Barui, J. Lin, W. Zhou, Y. Shen, N. Man, Y. J. Zhang, J. Xu, C. R. Patra and L. P. Wen, Biomaterials, 2014, 35, 899-907.
26 B. W. Festoff and B. A. Citron, Front. Neurol. Neurosci., 2019, 10, 59. 27 C. J. Reddel, C. W. Tan and V. M. Chen, Cancers, 2019, 11, 100.

28 C. Y. Tian, J. J. Xu and H. Y. Chen, Chem. Commun., 2012, 48, 8234-8236. 29 C. R. Patra, R. Bhattacharya, S. Patra, N. E. Vlahakis, A. Gabashvili, Y. Koltypin, A. Gedanken, P. Mukherjee and D. Mukhopadhyay, Adv. Mater., 2008, 20, 753-756.

30 H. M. Wang, Y. Fang, P. X. Yuan, A. J. Wang, X. L. Luo and J. J. Feng, Electrochim. Acta, 2019, 310, 195-202.

31 Y. J. Li, Y. Q. Li, N. Xu, J. H. Pan, T. F. Chen, Y. W. Chen and W. H. Gao, Sens. Actuators, B, 2017, 240, 742-748.

32 Y. Chen, B. Y. Jiang, Y. Xiang, Y. Q. Chai and R. Yuan, Chem. Commun., 2011, 47, 7758-7760.

33 J. Wang, Y. Shan, W. W. Zhao, J. J. Xu and H. Y. Chen, Anal. Chem., 2011, 83, 4004-4011. 